\title{
Tolerância ao fenantreno por fungos isolados de uma reserva ecológica de Mato
}

\section{Grosso, Brasil}

\author{
Tolerance to phenanthrene by fungi isolated from an ecological reserve of Mato Grosso, Brazil \\ Tolerancia al fentreno por hongos aislados de una reserva ecológica de Mato Grosso, Brasil
}

Recebido: 06/12/2021 | Revisado: 14/12/2021 | Aceito: 15/12/2021 | Publicado: 22/12/2021

Giovana Schneider

ORCID: https://orcid.org/0000-0003-1393-3996 Universidade do Estado de Mato Grosso, Brasil E-mail: giovana.schneider@unemat.br

Felipe Soares de Souza

ORCID: https://orcid.org/0000-0003-2955-670X Universidade do Estado de Mato Grosso, Brasil

E-mail: felipe.soares@unemat.br

João Arthur dos Santos Oliveira

ORCID: https://orcid.org/0000-0001-6864-1523

Universidade Estadual de Maringá, Brasil

E-mail: joaoarthur_oliveira@hotmail.com

Hilton Marcelo de Lima Souza

ORCID: https://orcid.org/0000-0001-9159-6847

Universidade do Estado de Mato Grosso, Brasil

E-mail: hilton.marcelo@unemat.br

\begin{abstract}
Resumo
A seleção de espécies com potencial em degradação de Hidrocarbonetos Policíclicos Aromáticos (HPAs) é uma etapa fundamental para o sucesso em programas de biorremediação. Entre alguns métodos, o teste de tolerância ao contaminante de interesse tem auxiliado os pesquisadores na obtenção de organismos com potencial em degradar HPAs. Dessa forma, o objetivo deste trabalho foi analisar o potencial de fungos, isolados de uma reserva ecológica do Centro-Oeste brasileiro, em tolerar fenantreno, um HPA de elevada toxicidade e encontrado em petróleo e seus derivados. Para tanto, discos da cultura dos fungos $(8 \mathrm{~mm})$, obtidos após 7 dias de crescimento em MEA 2\%, foram transferidos para placas de Petri contendo MEA 0,2\%, após inoculação superficial com cristais de fenantreno e incubados a $28^{\circ} \mathrm{C}$. O crescimento micelial foi medido por um período de 10 dias na ausência e presença de diferentes concentrações de fenantreno $(200 \mu \mathrm{g} / \mathrm{mL}, 600 \mu \mathrm{g} / \mathrm{mL}$ e $1000 \mu \mathrm{g} / \mathrm{mL})$, em triplicata. Os dados foram analisados através da taxa de crescimento fúngico (CF), inibição de crescimento fúngico (ICF) e Análise de Variância (ANOVA) seguida do Teste de Tukey ( $>>0,05)$. Ao décimo dia de crescimento, Phanerochaete australis SA18, Disporotrichum dimorphosporum SA09 e Lentinus crinitus SA37 se destacaram, com as maiores CF e baixa ICF, consequentemente. $P$. australis tolerou todas as diferentes concentrações de fenantreno e destacou-se estatisticamente dos demais fungos. A partir desse resultado, sugere-se novos estudos com $P$. australis, sobre produção enzimática, capacidade de degradação e produção de metabólitos de fenantreno.
\end{abstract}

Palavras-chave: Fungos; Biorremediação; Hidrocarbonetos policíclicos aromáticos.

\begin{abstract}
The selection of species with potential to degrade Polycyclic Aromatic Hydrocarbons (PAHs) is a fundamental step for the success of bioremediation programs. Among some methods, the contaminant tolerance test of interest has helped researchers to obtain organisms with the potential to degrade PAHs. Thus, the objective of this work was to analyze the potential of fungi, isolated from an ecological reserve in the Brazilian Midwest, to tolerate phenanthrene, a high toxicity HPA found in petroleum and its derivatives. For this purpose, fungus culture discs $(8 \mathrm{~mm})$, obtained after 7 days of growth in MEA 2\%, were transferred to Petri dishes containing MEA 0.2\%, after superficial inoculation with phenanthrene crystals and incubated at $28^{\circ} \mathrm{C}$. Mycelial growth was measured for a period of 10 days in the absence and presence of different concentrations of phenanthrene $(200 \mu \mathrm{g} / \mathrm{mL}, 600 \mu \mathrm{g} / \mathrm{mL}$ and $1000 \mu \mathrm{g} / \mathrm{mL})$, in triplicate. Data were analyzed using fungal growth rate (FC), fungal growth inhibition (FCI) and analysis of variance (ANOVA) followed by Tukey's test ( $\mathrm{p}>0.05)$. On the tenth day of growth, Phanerochaete australis SA18, Disporotrichum dimorphosporum SA09 and Lentinus crinitus SA37 stood out, with the highest CF and low ICF, consequently. P. australis tolerated all different concentrations of phenanthrene and stood out statistically from the other fungi. From these results, further studies with $P$. australis are suggested, on enzyme production, degradation capacity and production of phenanthrene metabolites.
\end{abstract}

Keywords: Fungi; Bioremediation; Polycyclic aromatic hydrocarbons. 


\begin{abstract}
Resumen
La selección de especies con potencial para degradar los Hidrocarburos Aromáticos Policíclicos (HAP) es un paso fundamental para el éxito de los programas de biorremediación. Entre algunos métodos, la prueba de tolerancia a contaminantes de interés ha ayudado a los investigadores a obtener organismos con el potencial de degradar los HAP. Así, el objetivo de este trabajo fue analizar el potencial de los hongos, aislados de una reserva ecológica en el Medio Oeste brasileño, para tolerar el fenantreno, un HPA de alta toxicidad que se encuentra en el petróleo y sus derivados. Para ello, los discos de cultivo de hongos $(8 \mathrm{~mm})$, obtenidos después de 7 días de crecimiento en MEA al $2 \%$, se transfirieron a placas de Petri que contenían MEA al 0,2\%, después de una inoculación superficial con cristales de fenantreno y se incubaron a $28^{\circ} \mathrm{C}$. Se midió el crecimiento micelial durante un período de 10 días en ausencia y presencia de diferentes concentraciones de fenantreno $(200 \mu \mathrm{g} / \mathrm{mL}, 600 \mu \mathrm{g} / \mathrm{mL}$ y $1000 \mu \mathrm{g} / \mathrm{mL})$, por triplicado. Los datos se analizaron utilizando la tasa de crecimiento fúngico (FC), la inhibición del crecimiento fúngico (FCI) y el análisis de varianza (ANOVA) seguido de la prueba de Tukey ( $>$ > 0,05). Al décimo día de crecimiento, se destacaron Phanerochaete australis SA18, Disporotrichum dimorphosporum SA09 y Lentinus crinitus SA37, con mayor FC y bajo ICF, consecuentemente. $P$. australis toleró todas las diferentes concentraciones de fenantreno y se destacó estadísticamente de los otros hongos. A partir de este resultado, se sugieren más estudios con $P$. australis, sobre la producción de enzimas, capacidad de degradación y producción de metabolitos de fenantreno.
\end{abstract}

Palabras clave: Hongos; Biorremediación; Hidrocarburos aromáticos policíclicos.

\title{
1. Introdução
}

Os Hidrocarbonetos Policíclicos Aromáticos (HPAs) são compostos orgânicos naturais que surgem através da combustão incompleta da matéria orgânica. Devido a presença de anéis aromáticos, as moléculas dos HPAs são muito complexas e estáveis, apresentam pouca afinidade com a água, se agregando em partículas orgânicas (Almeida, et al., 2007; Jacques, 2005; Jacques et al., 2007a).

Os HPAs que possuem dois ou três anéis aromáticos são classificados como de baixo peso molecular e apresentam elevada toxicidade. Já os que apresentam de quatro a seis, ou até sete anéis aromáticos são denominados como HPAs de alto peso molecular, sendo conhecidos por apresentar propriedades carcinogênicas (Celino et al., 2006; Jacques et al., 2007a; USEPA, 1993). Em temperatura ambiente os HPAs são sólidos, mas em altas temperaturas de fusão e ebulição alguns são semivoláteis, tendem a se acumular em tecidos lipídicos nos animais ou plantas, e podem permanecer por muitos anos no solo ou sedimentos. Por isso, são classificados como Poluentes Orgânicos Persistentes (POPs) (Caruso et al., 2008; Fulan, 2011; Jacques et al., 2007a).

Dentre os HPAs de baixo peso molecular, o fenantreno é conhecido por apresentar três anéis aromáticos, sendo encontrado no petróleo e seus derivados. Atividades como derramamentos acidentais, extração e uso de combustíveis fósseis, vazamentos em tanques de combustíveis subterrâneos, despejo de resíduos de motores veiculares, de oficinas mecânicas, garagens de automóveis, produtos asfálticos, entre outros, são alguns dos meios pelo qual ocorre a contaminação com fenantreno e outros HPAs no meio ambiente (Jacques et al., 2007b; Lima et al., 2017).

Entre os métodos empregadas para conter a contaminação ambiental, destaca-se a biorremediação, tanto pela sua eficiência, quanto pelo seu baixo custo. Além disso, a utilização mundial deste processo vem crescendo a cada dia (Jacques, 2005; Weber, 2013). As bactérias, fungos e algas são os principais organismos utilizados na biorremediação de HPAs. Os fungos, embora detenham de importantes habilidades, não têm recebido a atenção que merecem na biorremediação. A capacidade de formar extensas redes miceliais, baixa especificidade de suas enzimas, habilidade de usar poluentes como substratos e tolerar diferentes condições de estresse ambiental, são características que tornam os fungos promissores em estudos de recuperação ambiental de áreas contaminadas por diversos contaminantes ambientais (Harms et al., 2011; Lemos, 2008).

A maioria dos fungos degradadores de poluentes ambientais pertencem aos filos dos Ascomicetos e Basidiomicetos e os gêneros Aspergillus e Penicillium possuem muitas espécies que assimilam hidrocarbonetos. A literatura tem demonstrado que o grupo de fungos da podridão branca pertencente aos basidiomicetos, se destacam na biorremediação de HPAs, devido a 
presença das enzimas ligninolíticas, que são capazes de clivar os anéis aromáticos e minimizar sua toxicidade e até mineralizar esses compostos (Barbosa et al., 2008; Harms et al., 2011; Haritash et al., 2009; Salvi, 2011).

No entanto, para o uso racional de fungos para biorremediação, os estudos biológicos para seleção de cepas promissoras são primordiais. Os pesquisadores têm realizado diversos ensaios laboratoriais para a seleção de fungos com potencial em biodegradação de HPAs, tais como o teste de ácido gálico (reação de Banvendam) e guaiacol, a descoloração de corantes, tais como o Remazol Brilhante Blue R (RBBR), e o testes de tolerância ao contaminante de interesse (ArgumenoDilera et al., 2012; Lee et al., 2020; Lee et al., 2014; Santos, 2018; Silva, 2002; Silva et al., 2003; Souza, 2021; Souza, 2016; Souza et al., 2017; Wunch et al., 1997; Zafra et al., 2014). Altos índices de crescimento micelial e tolerância a certos níveis de contaminação são características consideradas como desejáveis em micro-organimos, sendo indicadores da habilidade de degradação de xenobióticos (Matsubara et al., 2006). A literatura tem demonstrado que pós a realização desses métodos citados acima, tem-se obtidos excelentes resultados em ensaios sobre biodegradação e produção de metabólitos de HPAs (Benguenab et al., 2020; Chulalaksananukul et al., 2006; Jacques, 2005; Lee et al., 2020; Passarini et al., 2011).

Diante da necessidade de estudos que fomentem o uso de diferentes organismos na recuperação de ambientes contaminados com HPAs, este trabalho teve como objetivo analisar o potencial de fungos isolados de uma Estação Ecológica do Estado de Mato Grosso em tolerar diferentes concentrações de fenantreno, no intuito de selecionar espécies promissoras para futuros estudos sobre biorremediação de solos contaminados com derivados do petróleo.

\section{Metodologia}

\subsection{Isolados fúngicos}

Os sete isolados de fungos utilizados neste trabalho foram coletados na Estação Ecológica da Serra das Araras, uma área de conservação e proteção integral gerida pelo Instituto Chico Mendes de Conservação da Biodiversidade (ICMBIO), vinculada ao Ministério do Meio Ambiente e integrada ao Sistema Nacional do Meio Ambiente (SISNAMA), localizada no

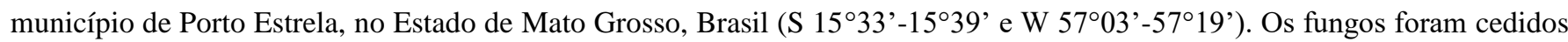
pelo Laboratório de Microbiologia do Centro de Pesquisas, Estudos e Desenvolvimento Agroambientais (CPEDA) da Universidade do Estado de Mato Grosso (UNEMAT), campus Universitário Professor Eugênio Carlos Stieler, Tangará da Serra.

Os fungos utilizados neste trabalho foram identificados por filogenia molecular por meio das regiões ITS1-5.8S-ITS2 (números de acessos OK393673 à OK393679), LSU (números de acessos OK383445 à OK383450) e $\beta$-Tubulina (número de acessos OK423453 à OK423454). Esses isolados foram selecionados pela capacidade de oxidação do ácido gálico e descoloração do corante RBBR, conforme descrito por Souza (2021). Os isolados foram reativados em meio MEA 2\% (20g/L extrato de malte, $15 \mathrm{~g} / \mathrm{L}$ Ágar) e incubadas por 10 dias a $28^{\circ} \mathrm{C}$.

\subsection{Avaliação da tolerância ao fenantreno}

Para realização dos ensaios foi preparada uma solução mãe com cristais de fenantreno (Sigma Aldrich, 98\%) $(50 \mathrm{mg} / \mathrm{mL})$ solubilizada em acetato de etila. Alíquotas dessa solução nas concentrações de $200 \mu \mathrm{g} / \mathrm{mL}, 600 \mu \mathrm{g} / \mathrm{mL}$ e 1000 $\mu \mathrm{g} / \mathrm{mL}$ foram aplicadas em uma placa de petri contendo meio de cultura MEA 0,2\% (2g de extrato de malte $+15 \mathrm{~g}$ de Ágar para $1 \mathrm{~L}$ de água destilada) e homogeneizadas no meio com auxílio de uma alça de drigalski. Placas apenas com meio MEA $0,2 \%$ constituíram o grupo controle.

Os fungos foram reativados inicialmente cultivando-os em meio de cultura MEA 2\% (20g de Extrato de Malte $+15 \mathrm{~g}$ de Ágar para $1 \mathrm{~L}$ de meio de água destilada) por 10 dias a $28^{\circ} \mathrm{C}$ e confirmação da pureza dos isolados (Figura 1). Logo após esse período, um disco de cada uma das respectivas culturas fúngicas $(8 \mathrm{~mm})$ foi utilizado nas placas contaminadas com 
fenantreno, colocando-o no centro de placas de Petri (triplicata) seguido do armazenamento em estufa do tipo B.O.D a $28{ }^{\circ} \mathrm{C}$ por 10 dias.

Figura 1: Fungos repicados em meio MEA 2\%. Lentinus crinitus SA37 (A), Cyathus bulleri SA16 (B), Polyporus sp. SA23 (C) e Phanerochaete australis SA18 (D).

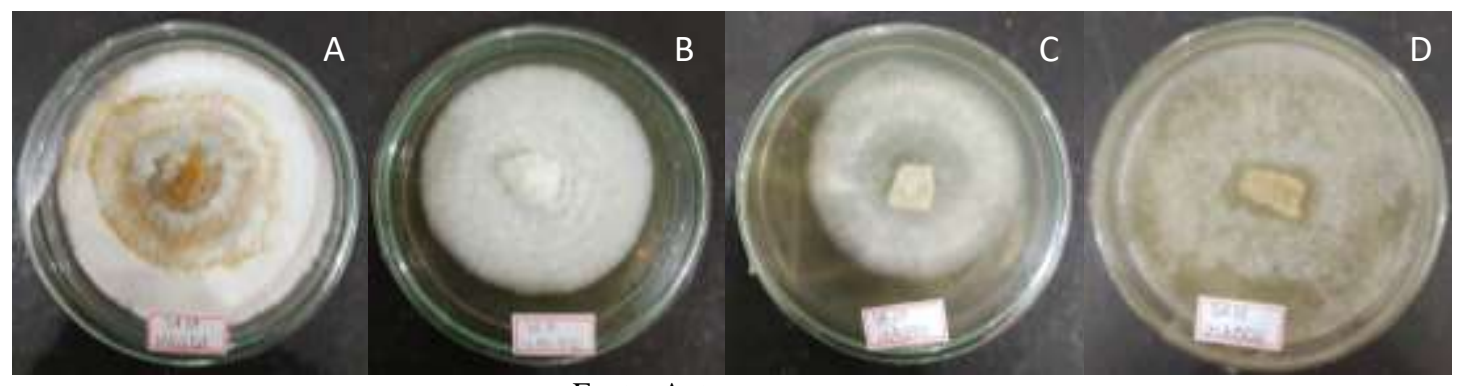

Fonte: Autores.

O crescimento radial do micélio fúngico foi medido diariamente, através de uma régua milimétrica. Para a medição, em cada uma das placas determinou-se o centro, onde foi inserido o bloco, e riscou-se três linhas, na parte inferior da placa, identificando as linhas 1, 2 e 3 para realização da medida média do halo de crescimento micelial (Figura 2). Depois dos 10 dias de medição, foi realizado o descarte do material em uma garrafa de vidro âmbar vedada e identificada, e encaminhada para descarte e incineração.

Figura 2: Retirada de blocos do repique (A) para o inóculo no ensaio biológico em triplicata (B), e posteriormente sua medição (C).

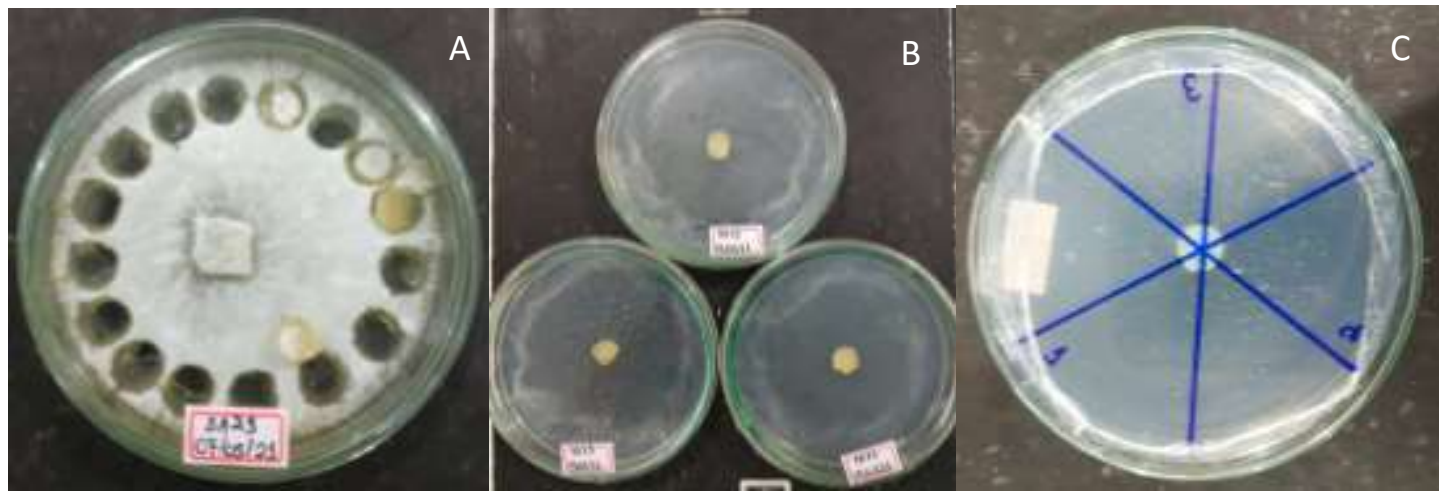

Fonte: Autores.

Para avaliar o crescimento micelial dos isolados foi realizado a taxa de crescimento fúngico $(\mathrm{CF})$, em que se calcula o diâmetro da colônia fúngica exposta ao fenantreno (DHPA) dividido pelo diâmetro da colônia do grupo controle (DC):

$$
\mathrm{CF}(\%)=(\mathrm{DHPA} / \mathrm{DC}) \times 100
$$

Também foi obtido a taxa de inibição do crescimento fúngico (ICF), calculado através da Taxa de crescimento fúngico, obtida no índice anterior, veja:

$$
\text { ICF }(\%)=100-\text { CF (Argumedo-Dilera et al., 2012) }
$$

Os dados foram avaliados no $5^{\circ}$ e $10^{\circ}$ dia após inóculo. Foi realizada uma análise estatística com dados de crescimento micelial médio através de Análise de Variância (ANOVA) seguida do Teste de Tukey (p>0,05). 


\section{Resultados}

Os resultados dos ensaios nos mostram diferentes respostas de crescimento dos fungos na presença do fenantreno. Ao $5^{\circ}$ dia, foi notável que a taxa de crescimento fúngico (CF) diminuiu conforme o aumento da concentração do contaminante. Phanerochaete australis SA18 obteve as maiores taxas de CF nas concentrações de $200 \mu \mathrm{g}(76,44 \%)$ e $600 \mu \mathrm{g}(56,22 \%)$, seguido de Hypoxylon fendleri SA41 (59,7\% e 49,25\%) e Polyporus sp. SA23 (56,15\% e 44,33\%). No entanto, nos ensaios com $1000 \mu \mathrm{g}$ de fenantreno, destacou-se Polyporus sp. SA23 (40,88\%) e H. fendleri SA41 (36,56\%) e menores taxas de inibição de crescimento fúngico (ICF) respectivamente $(59,12 \%$ e 63,44\%) (Figura 3AB). Consequentemente, as taxas de ICF nos confirma que, quanto maior a dosagem, maior a interferência do contaminante. Diaporthe mayteni SA07 apresentou menor tolerância em todos os tratamentos, apresentando altas taxas de ICF $(76,88 \%$ para o de $200 \mu \mathrm{g} / \mathrm{mL}, 83,57 \%$ para o $600 \mu \mathrm{g} / \mathrm{mL}$ e $88,02 \%$ para o de $1000 \mu \mathrm{g} / \mathrm{mL}$ ) (Figura 3B).

Figura 3: Taxa de crescimento fúngico (CF) do $5^{\circ}$ dia (A) e Taxa de Inibição do crescimento (ICF) do $5^{\circ}$ dia (B).
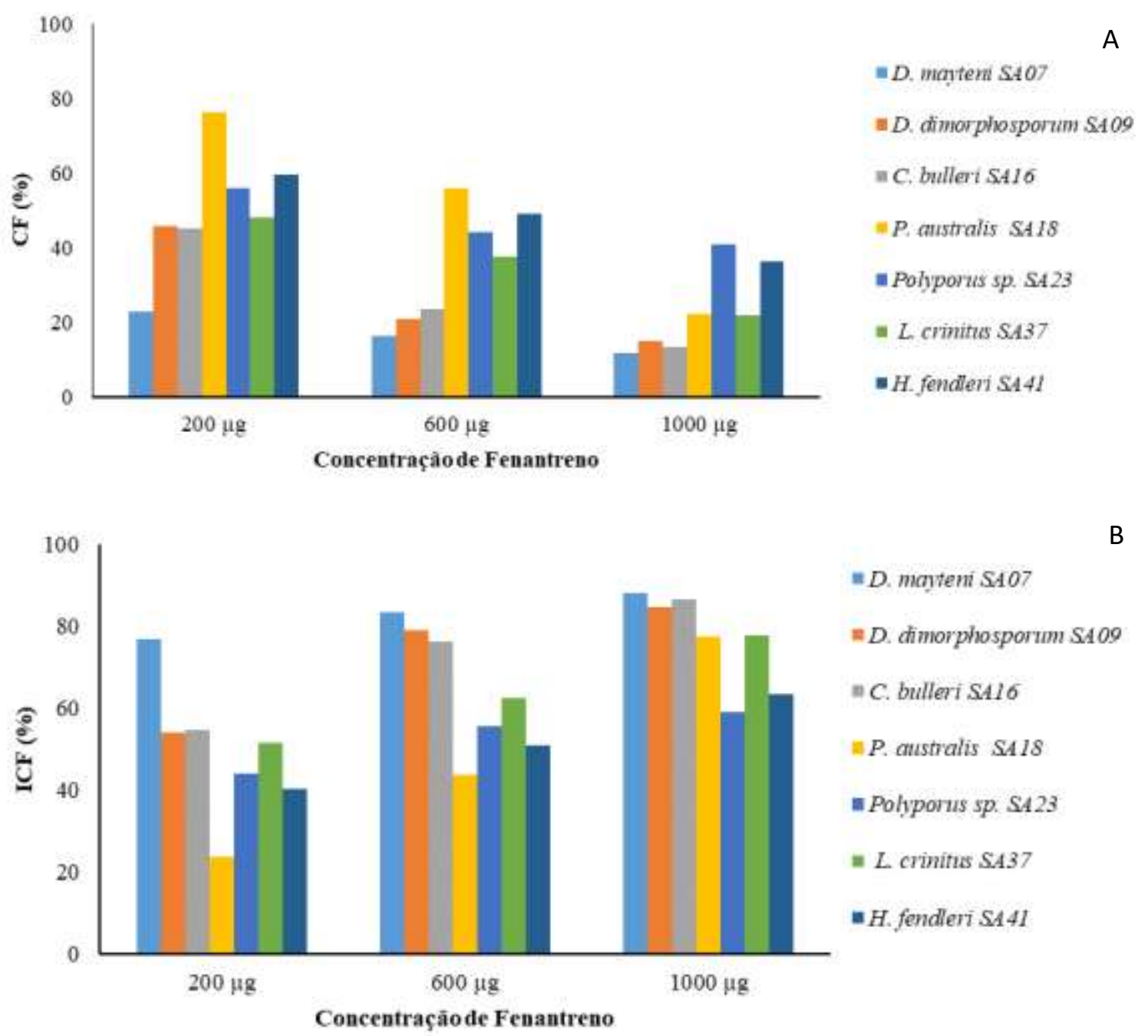

= D. manteni $S 407$

$=D$. dimorphosportum $\mathrm{SAOQ}$

NC. bulleri $\$ 416$

in. australis SA18

molyporus sp. S423

$=L$ crinitis $S .37$

H. fendleri $S A 41$

Fonte: Autores.

Podemos observar o crescimento médio dos micélios fúngicos ao $5^{\circ}$ dia através da Tabela 1 . Os fungos Disporotrichum dimorphosporum SA09 e P. australis SA18 obtiveram um crescimento de 45 mm no grupo controle, que equivale ao limite de crescimento da placa. O teste estatístico revela que $P$. australis SA18 apresentou o maior crescimento médio micelial em todas as diferentes concentrações de fenantreno $(34,4 \pm 0,19 \mathrm{~mm} ; 25,3 \pm 0,27$ mm; nos tratamentos de 200 
$\mu \mathrm{g} / \mathrm{mL}$ e $600 \mu \mathrm{g} / \mathrm{mL}$, respectivamente) $(\mathrm{p} \leq 0,05)$. A habilidade de crescimento dessa espécie é também observada no grupo controle, no entanto, o fato de continuar se destacando nas diferentes concentrações de fenantreno revela a habilidade em se desenvolver em meio a condições de estresse e toxicidade.

Tabela 1: Crescimento médio radial $(\mathrm{mm})$ do micélio no $5^{\circ}$ dia.

\begin{tabular}{cccccccc}
\hline & \multicolumn{7}{c}{ Fungo } \\
\hline & D. mayteni & D. dimorphosporum & C. bulleri & P. australis & Polyporus sp. & L. crinitus & H. fendleri \\
& SA07 & SA09 & SA16 & SA18 & SA23 & SA37 & SA41 \\
\hline controle & $35,9 \pm 0,23 \mathrm{c}$ & $45 \pm 0 \mathrm{a}$ & $18,5 \pm 0,05 \mathrm{e}$ & $45 \pm 0 \mathrm{a}$ & $20,3 \pm 0,02 \mathrm{~d}$ & $36,6 \pm 0,13 \mathrm{~b}$ & $13,4 \pm 0,03 \mathrm{f}$ \\
$\mathbf{2 0 0} \mathbf{\mu g}$ & $8,3 \pm 0,11 \mathrm{f}$ & $20,7 \pm 0,20 \mathrm{~b}$ & $8,4 \pm 0,05 \mathrm{e}$ & $34,4 \pm 0,19 \mathrm{a}$ & $11,4 \pm 0,02 \mathrm{~d}$ & $17,7 \pm 0,13 \mathrm{c}$ & $8 \pm 0,12 \mathrm{~g}$ \\
$\mathbf{6 0 0} \mathbf{~ g g}$ & $5,9 \pm 0,05 \mathrm{f}$ & $9,5 \pm 0,42 \mathrm{c}$ & $4,4 \pm 0,29 \mathrm{~g}$ & $25,3 \pm 0,27 \mathrm{a}$ & $9 \pm 0,02 \mathrm{~d}$ & $13,8 \pm 0,10 \mathrm{~b}$ & $6,6 \pm 0,09 \mathrm{e}$ \\
$\mathbf{1 0 0 0} \boldsymbol{\mu g}$ & $4,3 \pm 0,02 \mathrm{f}$ & $6,9 \pm 0,18 \mathrm{~d}$ & $2,5 \pm 0,33 \mathrm{~g}$ & $10,1 \pm 0,05 \mathrm{a}$ & $8,3 \pm 0,03 \mathrm{~b}$ & $8,1 \pm 0,19 \mathrm{c}$ & $4,9 \pm 0,01 \mathrm{e}$ \\
\hline & \multicolumn{7}{c}{ Fonte: Autores. }
\end{tabular}

No $10^{\circ}$ dia de ensaio também se observou uma diminuição da taxa de CF conforme aumento da dosagem de fenantreno. P. australis SA18 obteve as maiores taxas de crescimento nas concentrações de $200 \mu \mathrm{g} / \mathrm{mL}(100 \%), 600 \mu \mathrm{g} / \mathrm{mL}$ (100\%) e $1000 \mu \mathrm{g} / \mathrm{mL}$ (77,77\%), logo em seguida vem D. dimorphosporum SA09 (90,4\%, 87,55\% e 69,33\% respectivamente) e Lentinus crinitus SA37 (85,3\%, 77,55\% e 62,66\% respectivamente). Comparando com o resultado obtido ao $5^{\circ}$ dia, foi possível observar que Polyporus sp. SA23 e H. fendleri SA41 foram mais sensíveis a presença de fenantreno ao longo do tempo, pois suas taxas de CF diminuíram. Consequentemente, quanto maior a taxa de crescimento menor a taxa de ICF. $P$. australis SA18 atingiu uma taxa de ICF nula nos tratamentos de $200 \mu \mathrm{g} / \mathrm{mL}$ e $600 \mu \mathrm{g} / \mathrm{mL}$, e obtendo a menor taxa de ICF para o tratamento de $1000 \mu \mathrm{g} / \mathrm{mL}(22,23 \%)$ (Figura $4 \mathrm{AB})$. 
Figura 4: Taxa de crescimento fúngico $(\mathrm{CF})$ do $10^{\circ}$ dia (A) e Taxa de Inibição do crescimento (ICF) do $10^{\circ}$ dia (B).

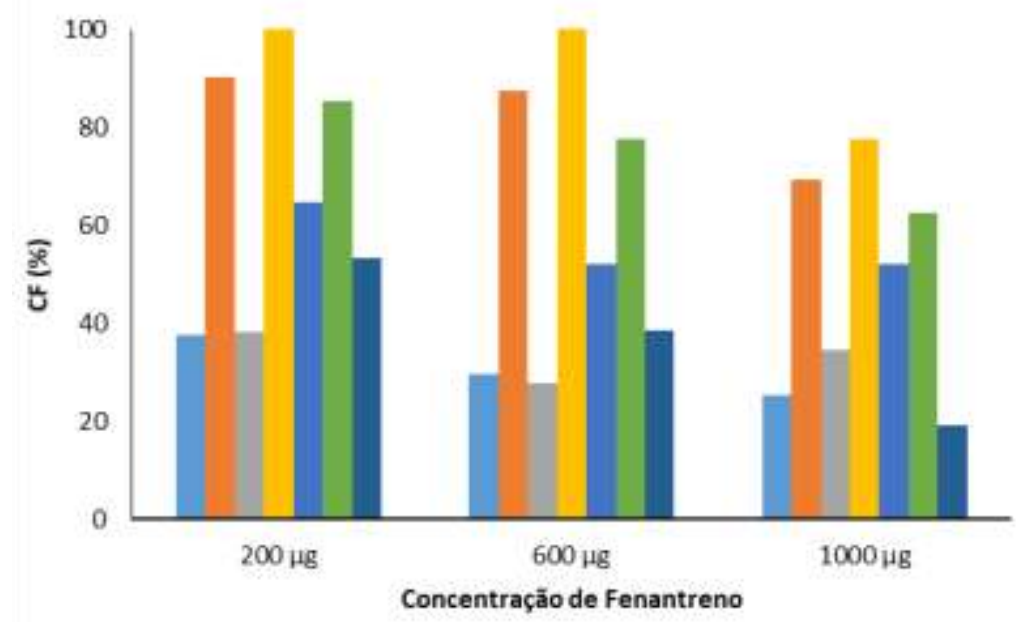

A

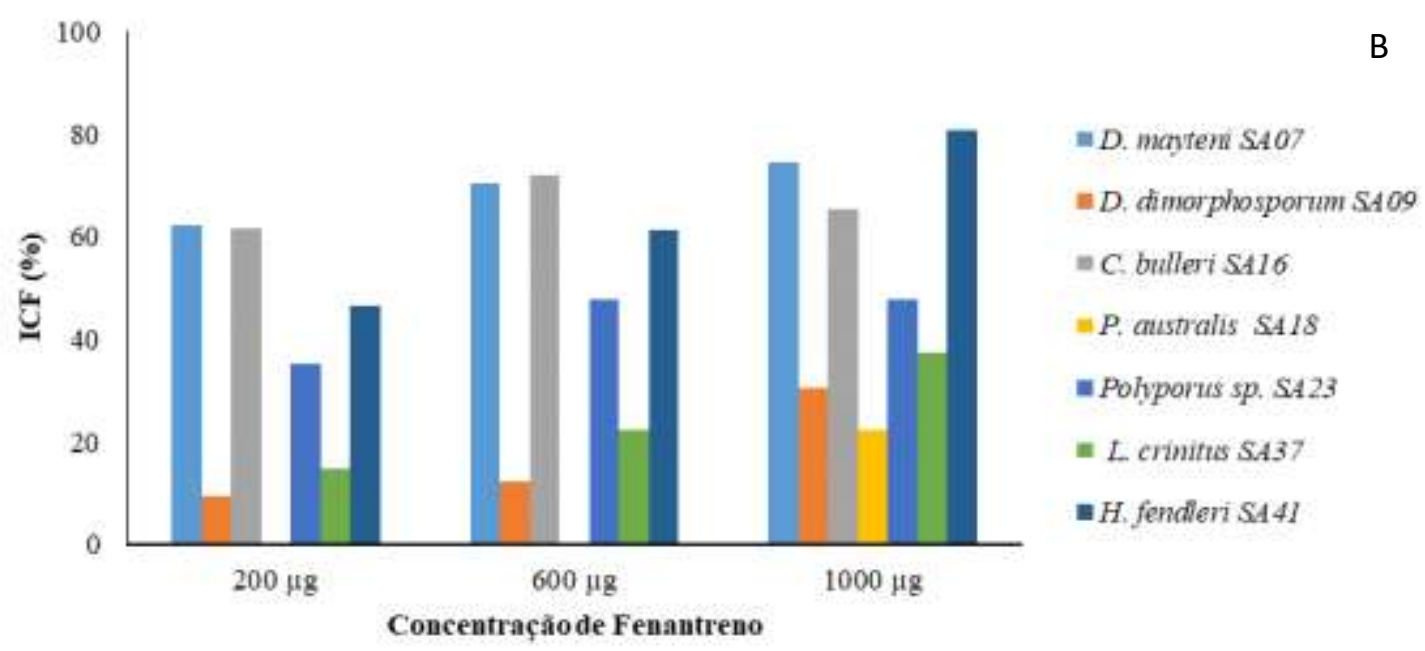

Fonte: Autores.

Os fungos D. dimorphosporum SA09 e Lentinus crinitus SA37 também obtiveram baixas taxas de ICF $(9,6 \%$ e $14,7 \%$ na concentração de $200 \mu \mathrm{g} / \mathrm{mL}, 12,45 \%$ e $22,45 \%$ para $600 \mu \mathrm{g} / \mathrm{mL}$ e $30,67 \%$ e 37,34\% para $1000 \mu \mathrm{g} / \mathrm{mL}$, respectivamente). Já os fungos que apresentaram as maiores taxas de ICF são o D. mayteni SA07 e Cyathus bulleri SA16, para os tratamentos de $200 \mu \mathrm{g} / \mathrm{mL}(62,36 \%$ e $61,63 \%)$ e $600 \mu \mathrm{g} / \mathrm{mL}(70,3 \%$ e $72,1 \%)$. Para o tratamento de 1000 $\mu \mathrm{g} / \mathrm{mL}$, quem obteve a taxa maior foi $H$. fendleri SA41 (80,94\%) (Figura 4AB).

Na Tabela 2 observa-se o crescimento médio micelial dos fungos no $10^{\circ}$ dia. $P$. australis SA18 obteve o maior crescimento médio radial entre os fungos em todos os tratamentos, $(200 \mu \mathrm{g} / \mathrm{mL}$ e $600 \mu \mathrm{g} / \mathrm{mL}$ com $45 \mathrm{~mm}$, e último tratamento obteve $35 \mathrm{~mm})$. Outro fungo que se destaca na análise estatística é o D. dimorphosporum SA09 (40,7 $\pm 0,57$ $\mathrm{mm}$ no tratamento de $200 \mu \mathrm{g} / \mathrm{mL}, 39,4 \pm 0,26 \mathrm{~mm}$ no tratamento com $600 \mu \mathrm{g} / \mathrm{mL}$ e 31,2 $\pm 0,4 \mathrm{~mm}$ no tratamento com $1000 \mu \mathrm{g} / \mathrm{mL})(\mathrm{p} \leq 0,05)$. 
Tabela 2: Crescimento médio radial $(\mathrm{mm})$ do micélio no $10^{\circ}$ dia.

\begin{tabular}{cccccccc}
\hline & \multicolumn{7}{c}{ Fungo } \\
\hline & D. mayteni & D. dimorphosporum & C. bulleri & P. australis & Polyporus sp. & L. crinitus & H. fendleri \\
& SA07 & SA09 & SA16 & SA18 & SA23 & SA37 & SA41 \\
\hline controle & $44,1 \pm 0,10 \mathrm{~b}$ & $45 \pm 0 \mathrm{a}$ & $34,4 \pm 0 \mathrm{c}$ & $45 \pm 0 \mathrm{a}$ & $44,1 \pm 0,10 \mathrm{~b}$ & $45 \pm 0 \mathrm{a}$ & $25,7 \pm 0 \mathrm{~d}$ \\
$\mathbf{2 0 0} \mathbf{\mu g}$ & $16,6 \pm 0,08 \mathrm{e}$ & $40,7 \pm 0,57 \mathrm{~b}$ & $13,2 \pm 0,11 \mathrm{~g}$ & $45 \pm 0 \mathrm{a}$ & $28,5 \pm 0,12 \mathrm{~d}$ & $38,4 \pm 0,21 \mathrm{c}$ & $13,7 \pm 0,11 \mathrm{f}$ \\
$\mathbf{6 0 0} \boldsymbol{\mu g}$ & $13,1 \pm 0,04 \mathrm{e}$ & $39,4 \pm 0,26 \mathrm{~b}$ & $9,6 \pm 0,59 \mathrm{~g}$ & $45 \pm 0 \mathrm{a}$ & $23 \pm 0,02 \mathrm{~d}$ & $34,9 \pm 0,21 \mathrm{c}$ & $9,9 \pm 0,03 \mathrm{f}$ \\
$\mathbf{1 0 0 0} \boldsymbol{\mu g}$ & $11,2 \pm 0,15 \mathrm{f}$ & $31,2 \pm 0,4 \mathrm{~b}$ & $11,9 \pm 0,21 \mathrm{e}$ & $35 \pm 0 \mathrm{a}$ & $23 \pm 0,11 \mathrm{~d}$ & $28,2 \pm 0,21 \mathrm{c}$ & $4,9 \pm 0,01 \mathrm{~g}$ \\
\hline
\end{tabular}

Fonte: Autores.

Observamos que tanto no $5^{\circ}$ dia quanto no $10^{\circ}$ dia quem se destaca através da análise estatística é o fungo P. australis SA18, obtendo as melhores taxas de crescimento fúngico (CF) e de crescimento médio radial do micélio, além de resultar nas menores taxas de inibição de crescimento fúngico (ICF), em que mesmo com o aumento da dosagem de fenantreno obteve bons resultados, o que indica sua capacidade de tolerar ambientes com elevadas concentrações de fenantreno e de estresse. $D$. dimorphosporum SA09 e L. crinitus SA37 mostraram dificuldade de crescimento ao $5^{\circ}$ dia analisado, porém ao $10^{\circ}$ dia alcançaram um bom desempenho na tolerância do fenantreno, ficando logo em seguida do P. australis (SA18).

\section{Discussão}

Os ensaios com fungos crescendo em um microambiente contaminado com fenantreno promovem o contato das hifas diretamente com um composto altamente tóxico, gerando estresse e alerta à sobrevivência. $\mathrm{O}$ Fenantreno $\left(\mathrm{C}_{14} \mathrm{H}_{10}\right)$ possui três anéis aromáticos organizados de forma não linear, alto poder hidrofóbico e capacidade de se adsorver a matéria orgânica (Usepa, 1993; Jacques, 2005; Furlan, 2011). Sua elevada estabilidade química acaba sendo uma forte barreira de impedimento ao desenvolvimento micelial.

A principal fonte do fenantreno é o petróleo e seus derivados. Nos componentes do petróleo há cerca de $30 \%$ de alcanos, 50\% de cicloalcanos, 15\% de aromáticos (UNEP, 1992). Os alifáticos, que não possuem ciclo ou anel em sua composição, estão entre os mais abundantes, e são mais facilmente degradados, já aqueles com maior estabilidade química e mais difíceis de serem degradados, os cíclicos, com cadeias fechadas, como o naftaleno e fenantreno, possuem dois e três anéis aromáticos, respectivamente (Bento, 2005; Lima, 2010). Assim, a capacidade do micélio fúngico em tolerar Hidrocarbonetos Policíclicos Aromáticos (HPAs) pode nos revelar o potencial desses organismos em degradar esses compostos.

Entre os diversos métodos indicados na literatura para selecionar espécies de fungos com potencial em biorremediação, os testes de tolerância têm sido utilizados por serem simples e de fácil realização. Geralmente, são realizados logo após análises qualitativas, tais como os testes de oxidação do ácido gálico/ guaiacol e descoloração do corante RBBR, que confirmam a presença de enzimas do complexo fenoloxidases. Diversos trabalhos têm apontado a importante dos testes de tolerância para seleção de fungos na biorremediação de HPAs. Argumeno-Dilera et al. (2012) realizou o teste de tolerância com diferentes concentrações de naftaleno, fenantreno e benzo[a]pireno em 11 cepas de Trichoderma (Ascomycota), demonstrando que o aumento das dosagens de HPAs possibilitaram maior inibição de crescimento micelial, além de destacar 4 cepas que foram altamente tolerantes a benzo[a]pireno, um HPA de alto peso molecular. Silva et al. (2003) isolou filamentos de fungos de sedimentos industriais puros e poluídos e avaliou o potencial dos mesmos em tolerar pireno e fenantreno, o qual demonstrou que $59 \%$ dos fungos avaliados foram tolerantes ao pireno e $30 \%$ ao fenantreno. Souza et al. (2017) também realizou a análise de tolerância de fungos filamentosos isolados de sedimentos contaminados por HPAs da região amazônica, no qual o fungo Hypoxylon sp. se destacou com a menor taxa de inibição de crescimento. Zafra et al. (2014) analisou a tolerância de isolados de fungos em consorcio de bactérias, com a finalidade de avaliar a tolerância em altas concentrações de 
fenantreno, pireno e benzo[a]pireno, obtendo resultados promissores.

Nesse trabalho, entre as espécies de fungos avaliadas destacam-se Phanerochaetae australis SA18, Disporotrichum dimorphosporum SA09 e Lentinus crinitus SA37, todos pertencentes a Filo Basidiomycota. Diversos trabalhos científicos têm demonstrado a habilidade de basidiomicetos na biodegradação de HPAs (Carvalho, 2010; Clemente et al., 2001; Jacques, 2005; Magrini, 2012; Santos, 2018; Silva, 2002; Silva et al., 2013; Souza et al., 2015; Souza et al., 2016; Wunch et al., 1997). Estes fungos são conhecidos por apresentarem um arcabouço enzimático capaz de realizar a oxidação e hidroxilação de compostos aromáticos e minimizar sua toxicidade no meio. Esse processo tem sido reportado pela ação das enzimas ligninolíticas extracelulares de baixa especificidade, tais como: lacase, lignina peroxidase, manganês peroxidase. A enzima intracelular citocromo P450 monooxigenase também detêm a mesma capacidade (Harms et al., 2011; Haritash et al., 2009; Salvi, 2011; Silva et al., 2003). Assim, como este grupo de fungos demonstrou destaque nessa pesquisa, sugere-se que há evidências de que a capacidade de tolerar fenantreno pode estar atrelada a produção de enzimas extracelulares e capacidade de degradar essa molécula.

A espécie Phanerochaetae australis apresentou os melhores resultados quanto a tolerância ao fenantreno. Esta espécie pertencente ao grupo dos fungos da podridão branca, do mesmo gênero do fungo Phanerochaete chrysosporium, que vem sendo muito citado na literatura como um bom degradador de HPAs de baixa e alta massa molecular (Bennet et al., 2002; Cerniglia, 1993; Dhawale et al., 1992; Harms et al., 2011; Silva et al., 2013), podendo levar a completa mineralização de compostos recalcitrantes (Bononi et al., 2008).

O fungo Hypoxylon fendleri SA41 que obteve um bom resultado para o $5^{\circ}$ dia, em contrapartida ao $10^{\circ}$ dia apresentou uma das mais altas taxas de inibição de crescimento, o que não corrobora com Souza et al. (2017) no qual em seu teste de tolerância o fungo Hypoxylon sp. mostrou taxas de inibição de crescimento mais baixas e uma maior tolerância ao fenantreno e ao pireno.

Para o fungo Disporotrichum dimorphosporum SA09, que obteve um destaque com uma taxa baixa de ICF, na literatura apenas há a indicação de habilidades biotecnológicas para a indústria alimentícia, por causa da produção de enzimas xilanases e glucanases, como em EFSA et al. (2020) que faz uma avaliação do uso das enzimas endo-1,4- $\beta$-xilanase e $\beta$ glucanase na indústria alimentícia. Essas enzimas são frequentemente usadas como aditivas para a eficiência nutritiva dos alimentos (Tachibana et al., 2010). Falta estudo para esse fungo, pois ele apresentou um bom resultado na análise de tolerância a fenantreno, indicando ter a presença de algum fator de interesse para biorremediação de HPAs.

O fungo Lentinus crinitus SA37 é basidiomiceto e tem sido citado na literatura pela capacidade de produzir a enzima lacase, o qual o torna importante para a pesquisa na biodegradação de HPAs, devido a capacidade oxidativa de anéis aromáticos dessa enzima (Neponucena, 2010; Almeida et al., 2018).

A próxima etapa da seleção de fungos com potencial para a biorremediação de HPAs é a realização de ensaios de biodegradação e análise quantitativa da produção enzimática em meio líquido, sendo necessária a realização destes testes com os fungos que obtiveram as maiores taxas de crescimento na presença do fenantreno, podendo assim contribuir na pesquisa sobre biorremediação de ambientes contaminados com HPAs.

\section{Conclusão}

Os fungos avaliados neste trabalho apresentaram certo grau de tolerância ao fenantreno, sendo que o fungo Phanerochaete australis SA18 apresentou as melhores taxas de CF e ICF, destacando-se estatisticamente, quanto a capacidade de crescimento micelial na presença de diferentes concentrações de fenantreno. Assim, sugere-se que novos trabalhos devem ser realizados com os fungos que foram indicados nesse trabalho para avaliação do seu potencial na biodegradação de fenantreno e outros HPAs, produção de enzimas ligninolíticas e formação de metabólitos que podem levar a mineralização do 
composto.

\section{Referências}

Almeida, F. V., Centeno, A. J., Bisinoti, M. C. \& Jardim, W. F. (2007, January 21). Substâncias tóxicas persistentes (STP) no Brasil. Química nova, 20(8), 1976-1985. https://doi.org/10.1590/S0100-40422007000800033

Almeida, P. H., Oliveira, A. C. C. De, Souza, G.R P. N. De, Friedrich, J. C., Linde, G. A., Colauto, N. B. \& Valle, J. S. Do. (2018). Decolorization of remazol brilliant blue R with laccase from Lentinus crinitus grown in agro-industrial by-products, 90(4), 3463-3473. https://doi.org/10.1590/0001-3765201820170458

Argumeno-Dilera, R., Alarcón, A., Ferrera-Cerrato, R., Almaraz, J. J. \& Peña-Cabriales, J. J. (2012). Tolerance and growth of 11 Trichoderma strains to crude oil, naphthalene, phenanthrene and benzo[a]pyrene. Journal of Environmental Management, 95, S291-S299. https://doi.org/10.1016/j.jenvman.2010.08.011

Barbosa, A. de M., Giese, E. C. \& Covizzi, L. G. (2008). Biodegradação de compostos aromáticos. Em I. S. De Melo \& J. L. De Azevedo (Eds.). Microbiologia ambiental (pp. 261-280). Jaguariúna, SP: Embrapa Meio Ambiente.

Benguenab, A. \& Chibani, A. (2020). Biodegradation of petroleum hydrocarbons by filamentous fungi (Aspergillus ustus and Purpureocillium lilacinum) isolated from used engine oil contaminated soil. Acta Ecologica Sinica, 41(5), 416-423. https://doi.org/10.1016/j.chnaes.2020.10.008

Bento, D. M. (2005). Análise Química da Degradação dos Hidrocarbonetos de Óleo Diesel no Estuário da Lagoa dos Patos [Dissertação de Mestrado em Oceanografia Física, Química e Geológica, Universidade Federal do Rio Grande]. Repositório Institucional FURG. http://repositorio.furg.br/handle/1/3526

Bononi, V. L. R., Machado, K. M. G., Matheus, D. R. \& Vitali, V. M. (2008). Biodegradação de Organoclorados no Solo por Basidiomicetos Lignocelulolíticos. Em I. S. De Melo \& J. L. De Azevedo (Eds.). Microbiologia ambiental (pp. 281-302). Jaguariúna, SP: Embrapa Meio Ambiente.

Costa, E. M. de M. B., Oliveira, V. De \& Pimenta, F. C. (2004). Citocromos P450 e Biotransformação Microbiana. Revista de Patologia Tropical, 33(1), 2131. https://doi.org/10.5216/rpt.v33i1.3121

Caruso, M. S. F. \& Alaburda, J. (2008). Hidrocarbonetos policíclicos aromáticos - benzo(a)pireno: uma revisão. Revista do Instituto Adolfo Lutz, 67(1), 1-27.

Carvalho, M. V. F. (2010). Avaliação química e toxicológica de solo contaminado por HPAs submetido à biodegradação pelo fungo basidiomiceto Pycnoporus sanguineus. [Dissertação de Mestrado em Biotecnologia, Universidade Estadual de Feira de Santana]. Coleção UEFS. http://tede2.uefs.br:8080/handle/tede/1157

Celino, J. J. \& Queiroz, A. F. de S. (2006). Fonte e grau da contaminação por hidrocarbonetos policíclicos aromáticos (HPAs) de baixa massa molecular em sedimentos da Baía de Todos os Santos. Revista Escola de Minas, 59(3), 265-270. https://doi.org/10.1590/S0370-44672006000300003.

Clemente, A. R., Anazawa, T. A. \& Durrant, L. R. (2001). Biodegradation of polycyclic aromatic hydrocarbons by soil fungi. Brazilian Journal of Microbiology, 32(4), 255-261. https://doi.org/10.1590/S1517-83822001000400001

Chulalaksananukul, S., Gadd, G. M., Sangvanich, P., Sihanonth, P., Piapukiew, J. \& Vangnai, A. S. (2006). Biodegradation of benzo(a)pyrene bya newly isolatedFusarium sp. FEMS Microbiology Letters, 262(1), 99-106. https://doi.org/10.1111/j.1574-6968.2006.00375.x

Dhawale, S. W., Dhawale, S. S. \& Dean-Ross, D. (1992). Degradation of phenanthrene by Phanerochaete chrysosporium occurs under ligninolytic as well as nonligninolytic conditions. Appl Environ Microbiol, 9(58), 3000-3006. https://doi.org/10.1128/AEM.58.9.3000-3006.1992

EFSA Panel on Food Contact Materials, Enzymes and Processing Aids (CEP), Silano, V., Baviera, J. M. B., Bolognesi, C., Cocconcelli, P. S., Crebelli, R., ... $\&$ Chesson, A. (2020). Safety evaluation of the food enzyme endo-1,4- $\beta$-xylanase and $\beta$-glucanase from Disporotrichum dimorphosporum strain DXL. EFSA Journal, 18(1). https://doi.org/10.2903/j.efsa.2020.5975

Furlan, B. (2011). Biodegradação de naftaleno, fenantreno e diesel por isolados do gênero Burkholderia da Amazônia [Dissertação de Mestrado em Microbiologia Aplicada, Universidade Estadual Paulista]. Repositório Institucional UNESP. http://hdl.handle.net/11449/95001

Harms, H., Schlosser, D. \& Wick, L. Y. (2011). Untapped potential: exploiting fungi in bioremediation of hazardous chemicals. Nature Reviews: Microbiology, 9(3), 177-192. https://doi.org/10.1038/nrmicro2519

Haritash, A.K. \& Kaushik, C. P. (2009). Biodegradation aspects of Polycyclic Aromatic Hydrocarbons (PAHs): A review. Journal of Hazardous Materials, 169(1-3), 1-15. https://doi.org/10.1016/j.jhazmat.2009.03.137

Jacques, R. J. S., Bento, F. M. \& Camargo, F. A. de O. (2007b). Biodegradação de hidrocarbonetos aromáticos policíclicos. Ciências e Natura, 29(1), 7-24. https://doi.org/10.5902/2179460X9736

Jacques, R. J. S. (2005). Biorremediação de Antraceno, Fenantreno e Pireno em um Argissolo [Tese de Doutorado em Ciências do solo, Universidade Federal do Rio Grande do Sul]. Repositório Digital UFRGS. http://hdl.handle.net/10183/5116

Jacques, R. J. S., Bento, F. M., Antoniolli, Z. I. \& Camargo, F. A. De O. (2007a) Biorremediação de solos contaminados com hidrocarbonetos aromáticos policiclicos. Ciência Rural, 37(4), 1192-1201. https://doi.org/10.1590/S0103-84782007000400049

Lee, A. H., Lee, H., Heo, Y. M., Lim, Y. W., Kim, C. M., Kim, G. H., \& Kim, J. J. (2020). A proposed stepwise screening framework for the selection of polycyclic aromatic hydrocarbon (PAH)-degrading white rot fungi. Bioprocess and Biosystems Engineering, 43, 767-783. https://doi.org/10.1007/s00449019-02272-w

Lee, H., Jang, Y., Choi, Y., Kim, M., Lee, J., Lee, H., \& Kim, J. (2014). Biotechnological procedures to select white rot fungi for the degradation of PAHs. Journal of Microbiological Methods, 97, 56-62. https://doi.org/10.1016/j.mimet.2013.12.007. 
Lemos, J. L. S., Barros, C. A., Oliveira, S. D., \& Reiche, A. P. (2008). Fungos filamentosos: agentes de degradação de petróleo e de hidrocarbonetos aromáticos policíclicos (HAPs). Série Tecnologia Ambiental, 16, 1-56.

Lima, D. C. R. DE. (2010). Microrganismos Degradadores de Petróleo Isolados de Solos Rizosféricos da Província Petrolífera de Urucu, Coari, Amazonas [Dissertação de Mestrado em Biotecnologia e Recursos Naturais, Universidade do Estado do Amazona]. Repositório Institucional UEA.

Lima, S. D. De, Oliveira, A. F. De, Golin, R., Caixeta, D. S., Lima, Z. M. De \& Morais, E. B. De. (2017). Gerenciamento de áreas contaminadas por postos de combustíveis em Cuiabá, Mato Grosso, Brasil. Revista Ambiente \& Água, 12(2), 299-315. https://doi.org/10.4136/ambi-agua.1872

Matsubara, M., Lynch, J. M., \& De Leij, F. A. A. M. (2006). A simple screening procedure for selecting fungi with potential for use in the bioremediation of contaminated land. Enzyme and Microbial Technology, 39(7), 1365-1372. https://doi.org/10.1016/j.enzmictec.2005.04.025

Magrini, M. J. (2012). Degradação de HPAs e Produção de Enzimas Ligninolíticas por Fungos Basidiomicetos Derivados de Esponjas Marinhas [Dissertação de Mestrado em Genética e Biologia Molecular, Universidade Estadual de Campinas]. BDTD.

Nepomucena, R. M. P. (2010). Avaliação do Potencial Microbiano de Crescimento e Secreção de Lacase do Fungo Amazônico Lentinus crinitus (L.ex Fr.) Fr [Dissertação de Mestrado em Biotcnologia e Recursos Naturais, Universidade do Estado do Amazonas]. Repositório Institucional UEA. http://repositorioinstitucional.uea.edu.br//handle/riuea/2216

Passarini, M. R.Z., Rodrigues, M. V.N., Silva, M. Da \& Sette, L. D. (2011). Marine-derived filamentous fungi and their potential application for polycyclic aromatic hydrocarbon bioremediation. Marine Pollution Bulletin, 62(2), 364-370. https://doi.org/10.1016/j.marpolbul.2010.10.003

Salvi, M. B. de. (2011). Fungos basidiomicetos em biorremediação [Tese de Doutorado em Biodiversidade Vegetal e Meio Ambiente, Instituto de Botânica de São Paulo].

Santos, V. S. dos. (2018). Seleção de basidiomicetos da região Amazônica com potencial para degradação de benzo(a)pireno [Dissertação de Mestrado em Biotecnologia e Recursos Naturais, Universidade do Estado do Amazonas]. Repositório Institucional UEA.

Silva, M.C.F., Antunes, A. A, Lins, C. I. M., Parente, A. H, Pereira, S. V. \& Campos-Takaki, G. M. (2013). Remoção do fenantreno por biomassa viva e inativada de Cunninghamella elegans ucp0542. E-xacta, 6(1), 1-8. http://dx.doi.org/10.18674/exacta.v6i1.839

Silva, M. da. (2002). Fungos filamentosos isolados de sedimentos estuarinos que metabolizam hidrocarbonetos aromáticos policíclicos [Tese de Doutorado em Ciências de Alimento, Universidade Estadual de Campinas]. Repositório Unicamp.

Silva, M. Da, Umbuzeiro, G. A., Pfenning, L. H., Canhos, V. P. \& Esposito, E. (2003). Filamentous Fungi Isolated from Estuarine Sediments Contaminated with Industrial Discharges. Soil and Sediment Contamination, 12(3), 345-356.

Souza, F. S. de. (2021). Seleção de Macrofungos Isolados da Estação Ecológica Serra das Araras/MT com Potencial para Degradação do Herbicida Glyphosate [Monografia de Graduação em Ciências Biológicas, Universidade Estadual de Mato Grosso].

Souza, H. M. De L., Barreto, L. R., Mota, A. J. Da, Oliveira, L. A. De, Barroso, H. Dos S. \& Zanotto, S. P. (2017). Tolerance to Polycyclic Aromatic Hydrocarbons (PAHs) by filamentous fungi isolated from contaminated sediment in the Amazon region. Acta Scientiarum. Biological Sciences, 39(4), 481488. https://doi.org/10.4025/actascibiolsci.v39i4.34709

Souza, H. M. De L., Oliveira, T. B. De, Rodrigues, A., Sette, L. D., Barroso, H. Dos S. \& Zanotto, S. P. (2015). Biodegradação de benzo(a)pireno por Fungos Filamentosos da Região Amazônica. Blucher Biochemistry Proceedings, 1(2), 271-274. 10.5151/biochem-vsimbbtec-22266

Souza, H. M. De L., Sette, L. D., Mota, A. J. Da, Neto, J. F. Do N., Oliveira, T. B. De, Oliveira, F. M. De, ... \& Zanotto, S. P. (2016). Filamentous Fungi Isolates of Contaminated Sediment in the Amazon Region with the Potential for Benzo(a)pyrene Degradation. Water Air Soil Pollut, 227(12), 431. https://doi.org/10.1007/s11270-016-3101-y

Tachibana, L., Pinto, L.G.Q., Gonçalves, G.S. \& Pezzato, L. E. (2010). Xilanase e $\beta$-glucanase na digestibilidade aparente de nutrientes do triticale pela Tilápia-do-nilo. Arquivo Brasileiro de Medicina Veterinária e Zootecnia, 62(2), 445-452. https://doi.org/10.1590/S0102-09352010000200026

Tortora, G. J., Funke, B. R. \& Case, C. L. (2017). Microbiologia (12a ed.). Artmed.

UNEP. (1992). Determination of petroleum hydrocarbons in sediments. United Nations Environment Programme.

Weber, B. D. \& Santos, A. A. (2013). Utilização da biorremediação como ferramenta para o controle da degradação. Engenharia Ambiental, $10(1)$, 114-133.

Wunch, K. G., Feibelman, T. \& Bennett, J. W. (1997). Screening for fungi capable of removing benzo[a]pyrene in culture. Applied Microbioly and Biotechnology, 47, 620-624. https://doi.org/10.1007/s002530050984.

Zafra, G., Absalón, Á. E., Cuevas, M. D. C. \& Cortés-Espinosa, D. V. (2014). Isolation and Selection of a Highly Tolerant Microbial Consortium with Potential for PAH Biodegradation from Heavy Crude Oil-Contaminated Soils. Water Air Soil Pollut, 225(1826), 1-18. https://doi.org/10.1007/s11270-0131826-4 